\title{
Flexibilität und Sicherheit auf dem Arbeitsmarkt: Zeit für eine erweiterte Analyse
}

Die Markteuphorie, die den Rückbau traditioneller Sicherungssysteme am Arbeitsmarkt begleitete, wird gegenwärtig abgelöst von der Wahrnehmung der Krisenanfälligkeit unseres Wirtschaftssystems. Die damit verbundene Skepsis führt deutlich weiter als es der Diskurs über Flexicurity zumeist thematisiert: Auf dem Weg zu einem realistischen Bild von den mehr oder weniger stabilen Balancen zwischen Flexibilität und Sicherheit in unterschiedlichen Gesellschaften müssen wir den Blick über die klassischen Institutionen wohlfahrtsstaatlicher Absicherung hinaus auf andere Bereiche des sozialen Lebens richten. Erst im Zusammenspiel der vielfältigen Einbettungen wirtschaftlichen Handelns können Regime der Governance von wirtschaftlicher Unsicherheit verstanden werden. ${ }^{1}$

\section{Einleitung}

Ökonomen und Politiker wurden durch die Finanzkrise 2007/2008 bei ihrer Suche nach dem vollkommen flexiblen Arbeitsmarkt gründlich aus der Bahn geworfen, denn es war und ist eine Krise im Kern des neoliberalen Modells der Marktsteuerung. Die für die Krise verantwortlichen Finanzmärkte des anglo-amerikanischen Typs waren bislang als reinster Ausdruck des Marktes angesehen worden - speziell in puncto zuverlässiger Vorausschau und Diskontierung zukünftiger Ereignisse durch kalkulierte Risikoteilung (Fama 1971; 1991). Weniger weit verbreitet ist die Erkenntnis, dass diese Märkte auch direkt relevant für die neoliberale Gestaltung des Arbeitsmarktes waren, geradezu charakteristisch für ein auf den Finanzmarkt gestütztes Modell der Kontrolle von Arbeitsmarktunsicherheit: Arbeitnehmern, die bislang ausschließlich von Leistungen des sozialen Sicherungssystems und dem Kündigungsschutz abhängig waren, um sich vor der Unbill des Arbeitsmarktes zu schützen, wurde nun die Möglichkeit eröffnet, unbesicherte Kredite aufzunehmen, vor allem Hypotheken. Die Risiken solcher Kredite für die Kreditgeber gingen durch extensiven Handel auf Sekundärmärkten zunächst quasi verloren. Die durch diese Kredite freigesetzte Kaufkraft förderte die wirtschaftliche Nachfrage und minderte dadurch die Unsicherheit. Sie wirkte ähnlich wie die keynesianische Nachfragesteuerung, allerdings durch reine Marktmechanismen ohne staatliche Eingriffe, in der Art eines "privatisierten Keynesianismus“ (Crouch 2009; Bellofiore/Halevi 2009).
Wir wissen jetzt, dass dieses Modell nicht nachhaltig war. Erstens ist es fraglich, ob das anglo-amerikanische Finanzmarktmodell die Kriterien des reinen Marktes überhaupt erfüllt, da es offensichtlich von Marktteilnehmern abhängt, die die versteckten Risiken in den von ihnen gehandelten Wertpapierbündeln nicht kennen. Dabei basiert der perfekte Markt in der Theorie doch stark auf rationalen Akteuren mit dem Streben nach umfassender Information. Zweitens hat die eilige Rettung des Bankensystems durch die Regierungen das System von staatlichen Eingriffen abhängig gemacht, die den Regeln des Marktes vollständig widersprechen. Das Modell scheint zu überleben, weil die involvierten Interessen zu mächtig sind, als dass man seinen Untergang riskieren könnte. Allerdings kann von einem reinen Markt oder einer marktförmigen Lösung im Umgang mit wirtschaftlichen Unwägbarkeiten keine Rede mehr sein. Es ist ein verzerrter Markt, der überdies von staatlichen Eingriffen und massiver finanzieller Unterstützung abhängig ist. Die Regierungen haben durch ihre Eingriffe „moral hazard” in das System eingeführt, d.h. die Versuchung der Banken zu risikoreichen Investitionen unter der Annahme, dass die Staatskasse für mögliche Verluste einspringt. $\mathrm{Ob}$ dies zu schlechteren Ergebnissen führt, als wenn man die Märkte ihrer eigenen Korrekturfähigkeit überlassen hätte, ist bisher nicht bekannt.

Die bereits bestehenden Zweifel an der Existenz reiner, sich selbst stabilisierender Arbeitsmärkte wurden von der Entwicklung in zweierlei Hinsicht verstärkt. Erstens, wenn das Finanzsystem nur mit Unterstützung von außen überleben kann, gilt das dann nicht auch für Arbeitsmärkte? Zweitens, wenn wie in einigen Ländern ungesicherte und flexible Arbeitsmärkte nur möglich waren, weil Arbeitnehmer unabhängig von ihren Arbeitseinkommen Zugang zu Krediten bekamen, was passiert dann nach dem Zusammenbruch der Märkte für zweitklassige Hypotheken und andere unbesicherte Kredite? Wenn dieses System die Antwort des Marktes auf die Unsicherheiten der Arbeitsmärkte war, war es insgesamt wirklich kostengünstiger und effizienter als die herkömmliche Sozialpolitik?

Zunächst ist es nötig, die Frage nach den Unsicherheiten auf dem Arbeitsmarkt und nach den optimalen Maßnahmen, die Schutzbedürfnisse der Arbeitnehmer mit der notwendigen Flexibilität in Einklang zu bringen, ganz neu zu stellen. Die Krise der unbesicherten Kredite (sog. SubprimeKrise) verhilft uns zu der Einsicht, dass nicht nur staatliche Arbeitsmarkt- und Sozialpolitik zur Bekämpfung wirtschaft-

\footnotetext{
1 Dieser Text ist Teil meiner Arbeit im Projekt „The Governance of Uncertainty and Sustainability: Tensions and Opportunities" (GUSTO, Fördernr. $225301 \mathrm{im}$ 7. Rahmenprogramm der Europäischen Union). Meinen Kollegen in diesem Projekt bin ich sehr dankbar für ihre vielen in den Text eingeflossenen Ideen.
}

Colin Crouch, Ph.D., ist Professor an der University von Warwick in Coventry und leitet das Institut für Governance und Public Management an der Warwick Business School. Arbeitsschwerpunkte: Gesellschaftsstrukturen in Europa, Arbeitsmarkt, Gender und Familie, Wirtschaftssoziologie, Neoinstitutionalismus. e-mail: Colin.Crouch@wbs.ac.uk Übersetzung aus dem Englischen: Yvonne Silber.

Deutsche Bearbeitung: Markus Promberger, Till Müller-Schoell. 
licher Unsicherheiten nötig ist, und dass unbesicherte Kreditvergabe nicht die einzige relevante Alternative ist. Dabei sind zumindest in einigen Ländern auch weitere Bereiche des sozialen Lebens von Bedeutung, die bislang nicht in den zentralen ideologischen Konflikt zwischen Markt und staatlicher Sozialpolitik geraten sind. Es gilt die Aufmerksamkeit für das jeweils spezifische Zusammenspiel einer Vielzahl gesellschaftlicher Institutionen zu schärfen, die für die Verarbeitung von Arbeitsmarktunsicherheiten sowie die Verteilung der Risiken einschlägig sind. Die folgenden Ausführungen liefern einen erweiterten Analyserahmen für die Untersuchung der Balancen zwischen Flexibilität und Sicherheit, der über die traditionell enger gefasste Arbeitsmarkt- und Sozialpolitik hinausgeht. Er erlaubt es, auf Dauer gestellte Regime gesellschaftlicher Unsicherheitsverarbeitung zu identifizieren sowie institutionelle Komplementaritäten bzw. Unverträglichkeiten zu erkennen.

\section{Ungewissheit, Sicherheit und Governance}

Die Ungewissheit über das zukünftige Marktgeschehen kann nicht durch die Herstellung von Gewissheit auf den Märkten aufgelöst werden. Erstere gehört zum Wesen des wirtschaftlichen Lebens. Die Akteure können versuchen, der Ungewissheit auf dem Markt durch Management - etwa Risikomanagement - zu begegnen, oder durch Schutzmaßnahmen, die außerhalb des Marktes liegen. Für diese außermarktlichen Begrenzungen bzw. Einbettungen sollte der Begriff „Sicherheit“ verwendet werden. Er meint z.B. soziale Sicherheit und Arbeitsmarktpolitik, Geschäftspraktiken der Unternehmen, Gewerkschaftsaktivitäten und das Handeln von Familien. Dabei bedeutet das Vorhalten verschiedener Formen von Sicherheit nicht, dass keine Risiken eingegangen werden. Es steht den Akteuren frei, Risiken einzugehen; wirtschaftliche Sicherheit schützt sie nur vor Risiken, die sie nicht selbst gewählt haben.

Individuen können ohne fremde Hilfe keine sichere Umgebung herstellen, da dies ein Minimum an kollektivem Handeln erfordert. Sehr reiche und mächtige Individuen lösen dieses Problem, indem sie eigene politische oder wirtschaftliche
Mittel einsetzen und so das kollektive Handeln zur Herstellung einer sicheren Umwelt kaufen oder anordnen. Die meisten Personen jedoch sind hierzu auf ihre Mitgliedschaft in Kollektiven angewiesen. Diese Kollektive, in denen Lebenschancen festgelegt werden, sind für jede Analyse von Sicherheit und Schutz von größter Bedeutung. Die wichtigsten sind internationale Organisationen, Nationalstaaten, andere lokale Herrschafts- und Mitgliedsverbände, Erwerbsorganisationen und Familien. Eine Studie über wirtschaftliche Sicherheit muss all diese Kollektive dahingehend betrachten, was sie tun, welche Bedeutung sie für das Leben des Einzelnen haben, und welche Beziehungen und Konflikte unter ihnen bestehen. Wenn sie eine Rolle dabei spielen sollen, Sicherheit auf verschiedenen Ebenen zuzuweisen, müssen sie über die Fähigkeit zu steuern, zu regieren verfügen, d.h. über Governance. Die GovernanceTheorie (Crouch 2006; Hollingsworth/ Boyer 1997; Hollingsworth et al. 2002) beschreibt verschiedene Governance-Modi wie Markt, Verband, Regierung etc. (siehe 3.1), die mit empirischen Einzelbeispielen kollektiven Handelns in Verbindung gebracht werden können.

\section{3 \\ Zentrale Begriffe für die Analyse nachhaltiger Sicherheit}

Um den engen Fokus der aktuellen staatlichen Politik in Sachen Flexibilität und Sicherheit zu erweitern, gilt es zunächst, ein Analyseschema zu entwickeln, das die ganze empirische Vielfalt von Politiken, Praktiken und Modi der Governance abdeckt. Eine vollständige oder theoretisch definierte Liste wird es hierfür allerdings nicht geben. Kreative Akteure suchen und finden ständig neue Möglichkeiten, um auf den fluktuierenden Weltmärkten Sicherheit zu erreichen oder Politiken abzuwandeln, die eigentlich für andere Zwecke bestimmt waren. Analytisch wenden wir die „Grammatik des Ungewissheits-Managements" an, d.h. die vier Hauptfragen: Wie? Wo? Wann? Zwischen wem? Die erste Frage bezieht sich auf die Modi der Governance, die folgenden drei darauf, wie der Schutz vor Ungewissheit verteilt wird.

\subsection{GOVERNANCE DER VERTEILUNG VON UNGEWISSHEIT}

Die Theorie benennt eine Reihe von Governance-Modi mit Schlüsselcharakter, von denen mindestens eines in jedem einzelnen Kollektivakteur gegenwärtig ist. Es sei angemerkt, dass diese Governance-Modi - Gesetze, Regierungen, Märkte, Unternehmenshierarchien, Verbände, Netzwerke und Gemeinschaften in der Praxis eines Handlungsfeldes oft verschränkt auftreten (Crouch 2006):

(1) Regierung ist selbstverständlich ein zentraler Modus der Governance im Feld von Beschäftigung und Sozialpolitik, einschließlich einiger erweiterter Aspekte. Hierunter fallen neben den nationalen auch regionale und lokale Regierungen und die Europäische Union. Gesetze und Regierung bilden gemeinsam die von der politischen Sphäre bereitgestellten Formen der Governance.

(2) Zudem gibt es zwei Governance-Modi, die der Wirtschaft zugehören. Der erste ist der Markt, ein Kollektiv, an dem praktisch jeder teilhat. Seine wichtigste Form des Managements von Ungewissheit besteht darin, die Ungewissheiten in handelbare Risiken umzuwandeln. Dabei verfügen die Marktteilnehmer über sehr ungleiche Ressourcen. Nicht nur die Marktmacht der Arbeitnehmer mit ihren unterschiedlichen Qualifikationen und Fähigkeiten bestimmen über den Erfolg ihrer Forderungen nach Sicherheitsgarantien von den Arbeitgebern, sondern der Markt selbst (zusammen mit Unternehmenshierarchien und fiskalischer Umverteilung) setzt Einkommensniveaus fest und ermöglicht Ansparen als häufigste Schutzform vor Ungewissheiten. Die Marktkräfte neigen zwar nicht automatisch dazu, Individuen zu kategorisieren, sie führen jedoch in Kombination mit anderen Formen der Governance (Regierungen, Unternehmenshierarchien) dazu, dass für Arbeitnehmer mit manuellen und nicht-manuellen Tätigkeiten oft unterschiedliche Pensions- und Krankheitsausfallzahlungen vereinbart werden.

(3) Unternehmenshierarchien verbinden sich mit Märkten und bauen auf ihnen auf, indem Unternehmen häufig unterschiedliche „Pakete“ von geregelten Ansprüchen für verschiedene Gruppen von Arbeitneh- 
mern etablieren, nicht nur für ihre direkt Beschäftigten, sondern auch für Leiharbeiter, Werkvertragsnehmer sowie Firmen in der Zulieferkette und deren Beschäftigte. Viele Regelungen in diesen Paketen haben direkten und großen Einfluss auf den Grad des Schutzes vor Ungewissheit, den ein Individuum erwarten kann. Erwerbsorganisationen können wichtige Gemeinschaften für den Einzelnen sein. Obwohl sie sich ausschließlich auf das Arbeitsleben beziehen, wirken daraus abgeleitetes Einkommen und Status auch auf die meisten anderen Lebensbereiche ein.

(4) Während in modernen Gesellschaften vor allem Politik und Wirtschaft die Quelle von Governance sind, werden verschiedene Bereiche des Wirtschaftslebens auch durch andere Institutionen reguliert und beeinflusst. Am stärksten formalisiert sind Verbände; besonders wichtig für das Handlungsfeld Arbeit sind sie durch die Vereinbarungen zwischen Gewerkschaften und Arbeitgeberverbänden oder einzelnen Unternehmen.

(5) Netzwerke als lockere, informelle Vereinigungsformen spielen in modernen Ökonomien eine wichtige Rolle, während für traditionelle Ökonomien erheblich engere informelle Gemeinschaften charakteristisch sind. Gemeinschaften lassen sich von Netzwerken unterscheiden durch die engere Kontrolle ihrer Mitglieder, die sich über viele Lebensbereiche erstreckt, und durch die Entwicklung moralischer Kodizes und Normen. In der Untersuchung der Steuerung von Sicherheit und Flexibilität haben die verschiedenen Gemeinschaften, besonders die Familie, eine hohe Bedeutung; Netzwerke hingegen sind vergleichsweise schwach. Lediglich manchmal werden solche Netzwerke relevant, in deren Rahmen Unternehmen ihre Arbeitsbeziehungen und lokale Zulieferketten untereinander formloser regeln als auf Verbandsebene üblich.

\subsection{DIE ÖRTLICHE, ZEITLICHE UND GRUPPENSPEZIFISCHE VERTEILUNG VON UNSICHERHEIT}

In Analysen der Inflation in den 1970er und 1980er Jahren erlangte die Theorie des kollektiven Handelns (Olson 1965) beträchtlichen Einfluss. Dies gilt insbesondere für Olson (1982), der zeigt, wie Unternehmensverbände und Gewerkschaften dazu neigen, Probleme durch Externalisierung auf Gruppen außerhalb ihres Einflussbereichs zu lösen. In Olsons Grenzfall der umfassenden Organisationen gelang es denjenigen Gruppen nicht, ihre Probleme auszulagern, deren Mitglieder die breite Mehrheit eines größeren Ganzen bildeten. Deshalb entwickelten sie Strategien, ihre Probleme zu lösen, ohne andere zu belasten. Obwohl die Verteilungsdynamik von Ungewissheiten sich von der Verteilungsdynamik einer Inflationssituation unterscheidet, ist das Thema "Auslagerung“ beiden gemeinsam. Denn beide betreffen Kollektive, die sich in Insider und Outsider aufteilen. In der Inflationsforschung wurde angenommen, dass die untersuchten Verbände innerhalb von Nationalstaaten existierten und dass der Anteil einer größeren sozialen Gruppe, der durch einen besonderen Verband repräsentiert wird, innerhalb dieses Nationalstaates gemessen werden kann. Relativiert man den Nationalstaat jedoch, wird die Analyse komplexer.

Innerhalb eines Kollektivs mit Insider/Outsider-Grenze eröffnen sich vier verschiedene Ansätze zum Management von Ungewissheit:

(1) Mitglieder könnten versuchen, ihre Ungewissheiten auf die gleiche Weise auf andere Kollektive auszulagern, wie es bereits mit der Inflation versucht wurde: örtliche Trennung als Antwort auf die Frage „Wo?".

(2) Ein ähnlicher Prozess könnte im Sinne einer zeitlichen Verlagerung in Gang gesetzt werden. Eine Gesellschaft von Menschen einer bestimmten Periode könnte - als Antwort auf die Frage „Wann?" - die Lösung verschiedener Probleme in die Zukunft verlagern und die Last späteren Generationen aufbürden. Dabei ist die Verortung in der Zeit erheblich komplexer als diejenige im Raum. Ein solcher Prozess vollzieht sich nur allmählich, da Menschen nicht nur mehrere Jahrzehnte leben, sondern sich auch in hohem Maße mit ihren Kindern und mindestens einer weiteren Generation identifizieren.

(3) Es kann sein, dass Mitglieder einer Gemeinschaft Ungewissheit akzeptieren und internalisieren, sie aber dabei durch Aufteilung im Rahmen kollektiver Maßnahmen minimieren müssen - als Antwort auf die Frage „zwischen wem?“.
(4) Wir müssen zudem der Idealvorstellung der Aufteilung von Ungewissheit „Zwischen wem?“ hinzufügen, dass Gemeinschaften in ihrem Inneren stratifiziert sein können und die Auslagerung so aussehen kann, dass die mächtigeren Mitglieder von den Schwächeren verlangen, einen unverhältnismäßigen Teil der Bürde der Ungewissheit zu tragen - gewissermaßen eine interne Auslagerung. Dies muss nicht notwendigerweise Ergebnis von bewusster Politik sein, sondern geschieht durch ständige Praxis. So treten Untergruppen in sozialen Gebilden hervor, die auf den ersten Blick als homogen erscheinen.

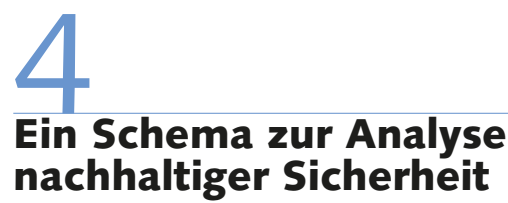

Nimmt man die dargestellten Dimensionen zusammen, in denen sich die Verarbeitung von Unsicherheit zwischen Gesellschaften unterscheidet, so ergibt sich ein Analyserahmen (Übersicht 1). Unterschiedliche Strategien und Praktiken der Governance und des Ausgleichs von Sicherheit und Flexibilität lassen sich in ihm entlang folgender drei Fragen beschreiben: Was sind die häufigsten Formen der angewandten Governance? Wie verhalten sie sich zu den Fragen von Ort, Zeit und interner Verteilung? Welche Formen von Auslagerung, Internalisierung oder interner Auslagerung kommen zum Einsatz? Damit ist der Grad der durch diese Strategie oder Praxis verursachten Ungleichheit angesprochen.

\subsection{ARBEITSRECHT}

Das Arbeitsrecht liefert den Rahmen für Arbeitnehmerrechte und deren Einschränkungen. Der Hauptzweck des Arbeitsrechts war - zumindest in demokratischen Zeiten - der Rechtsschutz der Beschäftigten gegenüber ihren Arbeitgebern, die auf den ersten Blick als mächtiger gelten als sie es tatsächlich sind (Davies/Freedland 2007; Knegt 2008). Das Arbeitsrecht stärkte daher die Sicherheit, in manchen Fällen auf Kosten der Flexibilität. In den letzten Jahren geriet es deswegen in andauernde Kritik vor allem von Ökonomen, da die Nachhaltigkeit von Beschäftigung als abhängig von wachsender Flexibilität angesehen wird. Ziel dieser Kritik war es, das 


\section{Übersicht 1: Potenzielles Analyseschema für Regimes der Verteilung von Ungewissheit}

\begin{tabular}{|c|c|c|c|c|}
\hline \multirow[t]{2}{*}{ Governancemodus } & \multirow[t]{2}{*}{ Politik und Praktiken } & \multicolumn{3}{|c|}{ Eigenschaften } \\
\hline & & $\begin{array}{l}\text { nachgordnete Gover- } \\
\text { nance-Modi }\end{array}$ & $\begin{array}{l}\text { Verteilung nach Ort, } \\
\text { Zeit, Gruppe }\end{array}$ & Externalisierung \\
\hline Gesetze & Arbeitsrecht & keine & interne Verteilung & Teilen bis interne Auslagerung \\
\hline \multirow[t]{4}{*}{ Überwiegend Staat } & Sozialpolitik über direkte Leistungen & Familie & interne Verteilung & Teilen \\
\hline & $\begin{array}{l}\text { Förderung der Qualifikation und } \\
\text { Beschäftigungsfähigkeit der Bevölkerung }\end{array}$ & Familie, Markt & Ort; interne Verteilung & $\begin{array}{l}\text { Auslagerung in Ökonomien mit geringerer } \\
\text { Qualifikation; interne Auslagerung, beim } \\
\text { Input an Familien mit Zugangsschwierig- } \\
\text { keiten, beim Output an Arbeitskraft mit } \\
\text { niedriger Bildungsstufe }\end{array}$ \\
\hline & $\begin{array}{l}\text { Öffentliche Förderung/ von } \\
\text { Branchen und Produktionsorten von } \\
\text { strategischer Bedeutung; öffentlicher } \\
\text { Beschäftigungssektor }\end{array}$ & $\begin{array}{l}\text { Unternehmens- } \\
\text { hierarchien }\end{array}$ & $\begin{array}{l}\text { Ort; auch interne } \\
\text { Verteilung }\end{array}$ & $\begin{array}{l}\text { Auslagerung in Ökonomien mit ähnlichen } \\
\text { Industrien, aber ohne Unterstützung; } \\
\text { intern: Teilen bis interne Auslagerung }\end{array}$ \\
\hline & Nachfragesteuerung durch den Staat & Markt & Zeit; interne Verteilung & Teilen \\
\hline $\begin{array}{l}\text { Staat, Markt, } \\
\text { Unternehmens- } \\
\text { hierarchien }\end{array}$ & Versicherungen und Pensionen & Verbände & interne Verteilung; Zeit & $\begin{array}{l}\text { Teilen innerhalb der Versichertengemein- } \\
\text { schaft; interne Auslagerung durch } \\
\text { Ungleichheiten beim Zugang zu } \\
\text { Auszahlungsplänen }\end{array}$ \\
\hline \multirow[t]{4}{*}{$\begin{array}{l}\text { Überwiegend } \\
\text { Markt }\end{array}$} & $\begin{array}{l}\text { Handelsbeziehungen zwischen } \\
\text { Nationalstaaten mit unterschiedlichen } \\
\text { Export- und Importbeziehungen }\end{array}$ & $\begin{array}{l}\text { Unternehmens- } \\
\text { hierarchien }\end{array}$ & Ort & $\begin{array}{l}\text { Auslagerung, Ungewissheit } \\
\text { konzentriert sich üblicherweise bei den } \\
\text { Exportökonomien }\end{array}$ \\
\hline & $\begin{array}{l}\text { Kreditvergabe zur Unterstützung des } \\
\text { Massenkonsums }\end{array}$ & unter Umständen Staat & Zeit; interne Verteilung & $\begin{array}{l}\text { Auslagerung in die Zukunft, wenn } \\
\text { Vertrauen kollabiert }\end{array}$ \\
\hline & $\begin{array}{l}\text { Ballungsräume einzelner } \\
\text { Wirtschaftszweige }\end{array}$ & Gemeinschaft & interne Verteilung & $\begin{array}{l}\text { Teilen bis interne Auslagerung, abhängig } \\
\text { von der Beziehung des Ballungsraums zum } \\
\text { Rest der Ökonomie }\end{array}$ \\
\hline & Schattenwirtschaft & Gemeinschaft & interne Verteilung & $\begin{array}{l}\text { interne Auslagerung, Ungewissheit } \\
\text { konzentriert sich bei den Personen in der } \\
\text { Schattenwirtschaft }\end{array}$ \\
\hline $\begin{array}{l}\text { Überwiegend } \\
\text { Unternehmens- } \\
\text { hierarchie }\end{array}$ & $\begin{array}{l}\text { Unternehmerisches Organisieren von } \\
\text { Aktivitäten mit unterschiedlichen } \\
\text { Sicherheitsgraden für verschiedene Länder } \\
\text { und Regionen; Zulieferketten }\end{array}$ & $\begin{array}{l}\text { Markt } \\
\text { Ort; interne Verteilung }\end{array}$ & $\begin{array}{l}\text { Auslagerung oder } \\
\text { interne Auslagerung in } \\
\text { Ökonomien/Regionen } \\
\text { an die Ränder der } \\
\text { Unternehmensstrategie }\end{array}$ & \\
\hline & $\begin{array}{l}\text { Interne Arbeitsmärkte und } \\
\text { Arbeitsorganisation }\end{array}$ & $\begin{array}{l}\text { Markt, Verbände, } \\
\text { Gemeinschaften }\end{array}$ & interne Verteilung & $\begin{array}{l}\text { interne Auslagerung in Randgruppen der } \\
\text { Beschäftigten/Leiharbeiter }\end{array}$ \\
\hline Verbände & Tarifverhandlungen & Markt & interne Verteilung; Ort & $\begin{array}{l}\text { Teilen bis interne Auslagerung, abhängig } \\
\text { von Inklusivität und Vereinbarungen; } \\
\text { eventuell Auslagerung in andere Länder } \\
\text { (Konkurrenz-Korporatismus) }\end{array}$ \\
\hline $\begin{array}{l}\text { Überwiegend } \\
\text { Gemeinschaft } \\
\text { (einschließlich } \\
\text { Familie) }\end{array}$ & $\begin{array}{l}\text { Transfers und Unterstützung zwischen } \\
\text { den Generationen }\end{array}$ & $\begin{array}{l}\text { Familie; womöglich } \\
\text { unterstützt durch } \\
\text { Regierung über } \\
\text { Sozialversicherungen }\end{array}$ & Zeit; interne Verteilung & $\begin{array}{l}\text { Teilen innerhalb der Familie; interne } \\
\text { Auslagerung an Familien mit geringen } \\
\text { Mitteln }\end{array}$ \\
\hline Verschiedene & $\begin{array}{l}\text { Protektion der besitzenden Eliten von den } \\
\text { Ungewissheiten des Arbeitsmarktes }\end{array}$ & verschiedene & interne Verteilung & $\begin{array}{l}\text { interne Auslagerung mit sehr ungleichen } \\
\text { Einsätzen und Auswirkungen }\end{array}$ \\
\hline
\end{tabular}

Arbeitsrecht in Richtung einer eigenen Rolle beim Ausgleich zwischen Sicherheit und Flexibilität zu bewegen. Eine wichtige Entwicklung in einigen Ländern war die Einführung der „reflexiven Regulation“ und gesetzlich herbeigeführte "freiwillige“ Regelungen zur Minderung der Schutzstandards. Hierzu passen die Versuche, in Tarifverhandlungen auf Unternehmensebene Abweichungen von Branchenstandards zu erreichen.

\subsection{SOZIALPOLITIK}

Eine der bedeutendsten öffentlichen Aufgaben ist das Erbringen von verschiedenen sozialen Dienstleistungen. Diese haben einen weitreichenden Einfluss auf die Sicherheit, nicht alle sind jedoch offensichtlich.
So verhindern direkt geleistete öffentliche Dienste den Marktzugriff auf dieses Handlungsfeld und sichern so in Zeiten wirtschaftlicher_Schwierigkeiten allen den Zugang zur Leistung. Vor allem bei geringer bezahlten Arbeitnehmern kann dies die Belastungen der unsicheren Arbeitsmarktlage lindern und sie womöglich eher zur Akzeptanz von größerer Marktungewissheit befähigen, als dies in Gesellschaften mit einem niedrigeren Niveau der öffentlichen Sozialdienstleistungen der Fall ist.

Daraus ergibt sich eine sekundäre, ursprünglich zufällige Konsequenz, die ihre eigenen Auswirkungen auf wirtschaftliche Ungewissheit hat. Öffentlich erbrachte Dienstleistungen umfassen eine Reihe von Betreuungsdienstleistungen: für Kinder, Kranke, Senioren. Werden diese Leistun- gen vom Markt bereitgestellt, sind sie für kleine Einkommensbezieher meist zu teuer, was zu einer Unterversorgung führt. Sie werden deshalb oft innerhalb der Familie geleistet, vor allem von Frauen. In diesem Fall wird die Leistung zwar erbracht, sie ist aber nicht Teil des Arbeitsmarktes. Wird die Leistung von staatlicher Seite angeboten oder subventioniert, wird sie immer noch überwiegend von Frauen erbracht, aber diesmal im Rahmen von Erwerbsbeteiligung. Damit werden Arbeitsplätze, Einkommen und Kaufkraft generiert. Darüber hinaus werden dadurch weitere Frauen von ihren innerfamiliären Betreuungsrollen befreit und in die Lage versetzt, anderen Erwerbstätigkeiten nachzugehen. Hieraus ergibt sich ein weiblicher Multiplikationseffekt auf die Beschäftigung. In 
denjenigen europäischen Ökonomien, die einen hohen Stand an öffentlichen Dienstleistungen aufweisen, ist sowohl der Anteil an weiblichen Erwerbstätigen als auch die Gesamtzahl der Beschäftigten höher als anderswo (Esping-Andersen 1999).

\subsection{STEIGERUNG VON QUALIFIKA- TION UND BESCHÄFTIGUNGS- FÄHIGKEIT}

Ein völlig mit freien Märkten kompatibles Mittel der Erzeugung von Sicherheit gegenüber künftigen Arbeitsmarktrisiken ist die Investition in die eigene Bildung. Dazu gehören auch Weiterbildung und Trainings, um sich gegen ungünstigen, das gegenwärtige Beschäftigungsverhältnis bedrohenden Wandel auf dem Arbeitsmarkt abzusichern. Während Wohlhabende dies aus eigener Kraft leisten können, ergibt sich hier ein weites Feld für beträchtliche staatliche Beteiligung, denn es besteht ein großes kollektives Interesse an der Weiterqualifizierung der Beschäftigten. Dieses Interesse geht über den individuellen Beschäftigten hinaus, denn für ihn ist es so gut wie unmöglich, Veränderungen in den Qualifikationsanforderungen des Arbeitsmarktes vorherzusehen.

Auch sozialpolitische Maßnahmen zur Förderung der Beschäftigung oder aktive Arbeitsmarktpolitik müssen hier berücksichtigt werden. In vielen Ländern werden Transferzahlungen zunehmend an Maßnahmen der aktiven Arbeitsmarktpolitik und im Gegenzug an öffentliche Förderung von Weiterbildung geknüpft. Zwischen Sozialversicherung und sozialer Sicherheit, aktiver Arbeitsmarktpolitik und individuellen Weiterbildungsanstrengungen besteht ein wichtiges Dreiecksverhältnis. In dem Ausmaß, in dem Transferzahlungen an eine Teilnahme in Maßnahmen der aktiven Arbeitsmarktpolitik gebunden sind, nehmen sie die Form einer „Workfare“-Bedrohung an und damit den Verlust der Leistungen, wenn die Förderungsgelegenheiten nicht wahrgenommen werden. Werden diese Maßnahmen mehr an einen verbesserten Zugang zu Investitionen in die eigene $\mathrm{Zu}$ kunft gebunden, sprechen wir eher von dänischer oder niederländischer „Flexicurity“ (Muffels et al. 2008; Rogowski 2008; Wilthagen 2002, 2004), wobei die Unterscheidung zwischen den beiden Begriffen alles andere als klar ist.

\subsection{STAATLICHE FÖRDERUNG EINZELNER WIRTSCHAFTSZWEIGE}

Ein breites Spektrum an kombinierten staatlichen und privaten Instrumenten versucht, die Arbeitsmarktsicherheit von Beschäftigten einzelner Branchen oder Regionen zu erhalten oder zu verbessern. Die klassischen Maßnahmen sind offener Protektionismus und die Förderung nationaler Champions. Letzteres ist allerdings in jüngerer Zeit unter heftige Kritik von Europäischer Union (EU) und Welthandelsorganisation (WTO) geraten, sodass dieses Instrument an Strahlkraft verliert.

Die branchenspezifischen Instrumente haben unterschiedliche Auswirkungen hinsichtlich Raum und interner Verteilung. Sie versuchen, Beschäftigung in einem Land auf Kosten eines anderen, das diese Instrumente (noch) nicht entwickelt hat, zu schützen oder zu fördern. Sie haben ähnliche Folgen auf Branchen und Regionen, die von der Politik weniger begünstigt werden, abhängig davon, ob zwischen den geförderten und den nicht geförderten Wirtschaftszweigen ein Nullsummenverhältnis, ein Multiplikator- oder ein Trickle-down-Effekt besteht.

\subsection{NACHFRAGESTEUERUNG}

Bei der keynesianischen Nachfragesteuerung handelt der Staat entlang der Marktentwicklung. Staatliche Ausgaben werden dazu genutzt, die Wirtschaft zu fördern, um Rezessionen zu vermeiden, und die Wirtschaft abzukühlen, wenn Inflation droht. Durch das Dämpfen von konjunkturellen Schwankungen soll das Maß von Unsicherheit auf dem Arbeitsmarkt verringert werden. Dieses Instrument fiel jedoch zunehmend der Kritik zum Opfer, in den 1970er Jahren die Inflationskrise verschärft zu haben und damit nicht mehr haltbar zu sein. Dennoch gehört es nach wie vor zu den gebräuchlichen Instrumentarien staatlicher Politik. Es funktioniert auf der Zeitachse. Mit seiner Hilfe lassen sich Staatsausgaben zur Abfederung von Konjunkturschwankungen nutzen, sein Einfluss auf die Gesellschaft ist in der Tendenz egalitär, allerdings nur dann, wenn die Staaten bereit sind, auf beiden Seiten des Konjunkturzyklus antizyklisch zu handeln und nicht nur bei Rezessionsgefahr die Nachfrage zu stimulieren.

\subsection{VERSICHERUNGEN}

Der Markt macht es möglich, Unsicherheiten Wahrscheinlichkeitswerte zuzuschreiben und sie in handelbare Risiken umzuwandeln, dies ist ebenfalls eine Form der Kostenverteilung von Ungewissheit auf der Zeitachse. In einer reinen Marktwirtschaft versichern sich auch Arbeitnehmer gegen Risiken, die ihre Sicherheit gefährden. So bedeutend das Versicherungsmodell jedoch für viele Bereiche sein mag, so ist es für die Gesamtheit der Arbeitnehmerschaft nicht sinnvoll, sich privat gegen Arbeitsmarktrisiken abzusichern. Hier griffen die Staaten mit Sozialversicherungen und sozialen Sicherungssystemen ein. In früheren Zeiten war die Steuerung von Sozialversicherungssystemen oft in verbandliche Governance-Strukturen eingebunden. Sie beschränken sich auf Umverteilungsfunktionen innerhalb einer gegebenen Risikopopulation, dabei operieren sie allerdings auf der Zeitachse, wie alle Versicherungen. Im Prinzip haben sie eine egalitäre Wirkung, wenn auch unterschiedliche Versicherungssysteme für verschiedene Berufsgruppen Ungleichheit bewirken. Ebenfalls bleiben viele Arbeitnehmer von jeglichen Versicherungen ausgeschlossen.

Eine aktivere Rolle spielt der Markt im Sozialversicherungssegment der Altersvorsorge. Diese stützt sich auf vier Säulen mit unterschiedlichen Governancestrukturen: die von der Regierung organisierte gesetzliche Rentenversicherung, von Verbänden organisierte Vorsorge in Ländern mit Pensionsfonds, die üblicherweise von $\mathrm{Ge}$ werkschaften und Arbeitgeberverbänden kontrolliert werden, betriebliche und berufsbezogene Altersvorsorge unabhängig von Verbändestrukturen und der private Altervorsorgemarkt.

\subsection{INTERNATIONALE HANDELS- BEZIEHUNGEN}

Bis hierher haben wir uns noch im Feld der gängigen Debatten um die Arbeitsmarktsicherheit bewegt. Wir wenden uns nun den weniger offensichtlichen, jedoch womöglich bedeutenderen Kontextfaktoren $\mathrm{zu}$, die das Niveau wirtschaftlicher Unsicherheit für verschiedene Beschäftigtengruppen bestimmen. Sicherheit kann innerhalb eines bestimmten lokalen oder regionalen Zusammenhangs gewährleistet werden, indem die Unsicherheit an 
andere Orte verschoben wird, die keine Möglichkeit haben, Gegenmaßnahmen zu ergreifen. Diese regionalen Unterschiede innerhalb der globalen Arbeitsteilung verdienen fundamentale Berücksichtigung bei allen Versuchen, nationale Sozial- oder Wohlfahrtsstaatsmodelle zu typisieren, werden gewöhnlich jedoch komplett ignoriert. Gängige Typisierungsversuche behandeln die verschiedenen Länder als mehr oder weniger gleiche Einheiten, als Beispiele desselben Phänomens. Wenn wir allerdings anerkennen, dass Länder keine geschlossenen Einheiten sind, sondern Knoten in einem hierarchisierten Netzwerk (wie in der Weltsystemtheorie, Wallerstein [1986]), die ganz unterschiedliche Positionen in diesem Netzwerk besetzen, ändert sich die Art der Analyse erheblich.

Wenn die Arbeitnehmerschaft eines Landes auf Exportmärkte spezialisiert ist, hängt ihre Sicherheit erheblich von den Fluktuationen im Welthandel ab - es sei denn, die betroffenen Exportmärkte sind wenig elastisch, was eine sehr günstige Position zur Folge haben kann - wie etwa nach wie vor die deutsche Wirtschaft, das beste Beispiel dieses Ansatzes in der entwickelten Welt. Damit einher geht eine Zurückhaltung bei der Wirtschaftsförderung durch Binnennachfrage. Dies hat in Deutschland in den letzten Jahren zu relativ niedrigen Standards bei öffentlichen und privaten Dienstleistungen geführt und damit auch zu einem wenig entwickelten weiblichen Multiplikatormarkt.

\subsection{KREDITBASIERTE ÖKONOMIEN}

In einigen Ländern hat sich in den letzten Jahren eine Marktpraxis entwickelt, die das individuelle Konsumverhalten vom Arbeitseinkommen durch die ausgedehnte Vergabe unbesicherter Kredite abgekoppelt hat, gewöhnlich durch Hypotheken, aber auch durch Kreditkarten. Obwohl diese Praxis ausschließlich für das Profitstreben der Finanzmärkte entwickelt wurde, zeigte sie die unerwartete Wirkung einer Stressminderung bei der Sorge über die eigene Sicherheit auf dem Arbeitsmarkt. Die Praxis entstand vor allem in den USA, dem Vereinigten Königreich, Island und Irland; sie lässt sich - wie oben eingeführt - als „privatisierter Keynesianismus" bezeichnen. Das Modell funktioniert nur bei einer stetigen Aufwärtsbewegung auf dem Immobilienmarkt, eine Situation, die sich durch Regierungseingriffe garantie- ren lässt. Diese Form von marktbasierter Governance ist allerdings anfällig für Vertrauensverluste in die gehandelten Werte, wie sich 2007-2008 in der weltweiten Finanzkrise zeigte.

\subsection{WIRTSCHAFTSCLUSTER}

Eine Sonderform marktorientierter Beschäftigungssicherung findet sich in Industrieregionen und anderen Gebieten mit lokaler wirtschaftlicher Spezialisierung, in denen sich viele Unternehmen konzentrieren, insbesondere, jedoch nicht ausschließlich, kleine und mittlere Unternehmen (KMU), die sich gegenseitig zuarbeiten. Hier sind vor allem die Industriezentren in Italien zu nennen (Pyke et al. 1990), in Dänemark (Kristensen et al. 1999) und in Kalifornien (Kenney 2000). Solche Wirtschaftscluster erzeugen mehr soziale Sicherheit als ein einzelnes Unternehmen. Arbeitnehmer erfahren eine Vielfalt an verfügbaren Beschäftigungsmöglichkeiten in einem geografischen Gebiet und in sozialen Netzwerken. Die Unsicherheit eines einzelnen Unternehmens stellt deshalb nicht notwendigerweise eine Bedrohung für das Beschäftigungs- und Einkommensniveau des betroffenen Wirtschaftszweiges oder der Region dar.

\subsection{SCHATTENWIRTSCHAFT}

Manchmal sind Wirtschaftscluster Teil der Schattenwirtschaft, auch wenn dieser Begriff wesentlich weiter zu fassen ist. Die meisten Studien über Beschäftigungsschutzmaßnahmen und Flexibilität befassen sich mit den offiziellen Regelungen und gehen davon aus, dass diese wirkungsvoll eingesetzt werden. Damit gehen sie an den Tatsachen der Arbeitsmarktregulierung und einer großen Zahl von Arbeitnehmern vorbei. Die Schattenwirtschaft erscheint schließlich in keiner Statistik (Buehn/Schneider 2007). Die Forschung sollte sich mit dem vollen Ausmaß von Größe und Lokalisierung dieser Wirtschaftsform beschäftigen, denn sie stellt ein wirksames Instrument zur Ausgrenzung von Arbeitskräften dar, da die Schattenarbeiter normalerweise von keinerlei formalen Sicherungsmaßnahmen der regulären Belegschaften profitieren. Ungewissheit lässt sich daher auf diese Arbeitskräfte auslagern und fördert damit eine gewisse Flexibilität.

\subsection{MANAGEMENTHANDELN IN EINZELNEN FIRMEN}

Unternehmenshierarchien großer Firmen beeinflussen - neben dem Markt - die räumliche Verteilung von Sicherheit, indem sie Arbeitsplätze mit unterschiedlichen Sicherheitsgraden in verschiedenen Teilen der Welt oder Regionen eines großen Nationalstaats platzieren. Management und Politik des einzelnen Unternehmens ist gemeinsam mit anderen Arten von Governance verantwortlich für das Entstehen unterschiedlicher Sicherungsstufen für die Beschäftigten - etwa durch die Definition unterschiedlicher Typen von Arbeit und zugehöriger Privilegien. Dabei erstreckt sich der Einfluss der internationalen und auch der internen Praktiken von der firmeneigenen Belegschaft bis hinein in die Zuliefererkette. Managementstrategien zielen auf maximale Durchsetzung der jeweiligen Firmeninteressen, die geografische Verteilung von unterschiedlichen Sicherungsgraden fällt dabei lediglich als Nebenprodukt an. Deren soziale Auswirkungen und die daraus resultierenden Ungleichheiten können jedoch immens sein.

\subsection{KOLLEKTIVE ARBEITS- BEZIEHUNGEN}

Verbändebasierte Governance bzw. Korporatismus, besonders Tarifverhandlungen zwischen Gewerkschaften und einzelnen Unternehmen oder Unternehmensverbänden, werden üblicherweise als positiv für die Sicherheit auf dem Arbeitsmarkt wahrgenommen. Gleichzeitig wird jedoch kritisiert, dass diese Sicherheit durch verminderte Flexibilität erkauft wird und damit untragbar ist. Ein Gleichgewicht von Sicherheit und Flexibilität lässt sich alternativ auch durch Differenzierung in Insider und Outsider herstellen. Da Tarifverhandlungen auf strategischer Basis stattfinden, kann hier auch über Flexibilität und Sicherheit in unterschiedlichen, jedoch nicht allen möglichen Zusammenhängen verhandelt werden. So werden z.B. die Arbeitnehmervertreter bei Tarifverhandlungen mit Einzelunternehmen eventuell beim kurzfristigen Schutz ihrer Mitglieder gegenüber möglichen Bedürfnissen des Unternehmens nach Flexibilität nachgeben müssen, wenn es um das Überleben und den Erfolg des Unternehmens geht, was man "concession bargaining" nennt. Alternativ dazu werden Gewerk- 
schaften die Positionen der gegenwärtigen Insider zulasten der Outsider schützen, etwa durch Faustregeln wie „first in, last out" oder Seniorität - die zuerst eingestellten Arbeitnehmer werden als Letzte entlassen, was auf eine Diskriminierung junger Arbeitnehmer hinauslaufen kann - oder durch die Unterteilung der Belegschaft in Stammarbeitnehmer und Zeitarbeitnehmer. Wirtschaftswissenschaftliche Theorien halten diese Praktiken für grundlegend bei den Gewerkschaften (z.B. Blanchard/Summers 1986; Rueda 2005, 2007), weil sie ein unternehmensgestütztes Verhandlungssystem wie in USA und Japan unterstellen. Gewerkschaften jedoch, die Mitglieder einer ganzen Industrie oder eines anderen Teilarbeitsmarktes vertreten, werden solche Vereinbarungen eher als Gefahr dafür sehen, dass die Arbeitgeberseite in Zukunft eher zu befristeten und ungesicherten als zu stabilen Verträgen tendieren wird. So sind etwa in Spanien, dem europäischen Land mit den meisten befristeten Arbeitsverträgen, die Gewerkschaften entschieden gegen diese Strategie (Talani/Cerviño 2003).

In Tarifverhandlungen auf der Ebene oberhalb des Einzelunternehmens ist das Flexibilitäts-/Sicherheitsverhältnis oft Verhandlungsgegenstand, allerdings nur dann, wenn Gewerkschaften und Arbeitnehmerverbände koordiniert vorgehen und sie sich von ihrer Struktur her der Verantwortung für die makroökonomischen Folgen ihrer Aktivitäten nicht entziehen können und darüber hinaus für den betroffenen Wirtschaftszweig oder die Ökonomie eine besondere Rolle spielen (Traxler 2003; Traxler et al. 2001; 2008), ähnlich wie bei den Antiinflationsstrategien der 1970er Jahre.

\subsection{TRANSFERS UND UNTER- STÜTZUNG ZWISCHEN DEN GENERATIONEN}

Die Familie als Institution außerhalb des Marktes spielt eine wichtige Rolle für das Ausbalancieren von Sicherheit zwischen ihren Mitgliedern und über die Zeit. Sie ist ein wichtiger Kanal für generationenübergreifende finanzielle Transfers, z.B. bei der Hausfinanzierung. Einzelne Elemente dieser Funktionen lassen sich zwar in den meisten Gesellschaften beobachten, jedoch sind ausgeprägte Differenzen festzustellen. So bestehen bemerkenswerte Unterschiede beim Durchschnittsalter junger Leute, die das Elternhaus verlassen; sie reichen von den frühen 20ern in Nordwesteuropa bis über 30 in Südwesteuropa. Dies hat Auswirkungen darauf, wie jungen Leuten in schwierigen Arbeitsmarktsituationen geholfen wird. Soziale Normen über familiäre Verpflichtungen tragen zur Bestimmung dieser Differenzen bei, manchmal mit Unterstützung der Sozial- und Steuerpolitik (Jurado Guerrero 1999). Wenn man Gleichaltrige betrachtet, hängen diese Mechanismen vom unterschiedlichen Zugang der einzelnen Familien zu den relevanten Ressourcen ab.

\subsection{GESCHÜTZTE ELITEN}

Die historisch wichtigste Form der Verteilung von Sicherheit bestand stets in einer Kombination verschiedener GovernanceModi: Markt, Staat, Gesetze, Gemeinschaften. Das bedeutet, dass privilegierte Minderheiten einer Bevölkerung wirtschaftlichen Schutz durch Unabhängigkeit von den Fluktuationen auf den Arbeitsund Gütermärkten erreichen - typischerweise Adlige, Grundrentenbesitzer sowie andere hoch vermögende Personen. Diese Privilegien entstehen durch die verschiedensten Kombinationen politischer und marktwirtschaftlicher Entscheidungen. In solchen Gesellschaften konzentriert sich der Konsum in hohem Maße innerhalb dieser Gruppen. Bevor sich die Verbrauchermärkte entwickelten, herrschte dieses System in vielen Gesellschaften vor, z.B. in den meisten vorindustriellen Gesellschaften und solchen im frühen Stadium der Industrialisierung (wie im heutigen China). Teilweise wurden diese privilegierten Positionen von der kapitalistischen Wirtschaftsweise mit Eintritt in die fordistische Massenproduktion im 20. Jahrhundert beseitigt. Dennoch haben privilegierte Gruppen üblicherweise besseren Zugang zu den politischen Entscheidungsträgern als die Mehrheit der Bevölkerung.

Dies kann auch für die besitzlosen Minderheiten der modernen Arbeitnehmerschaft gelten, wie schon oben im Zusammenhang mit Schattenwirtschaft und Klassifikation des Arbeitsmarkts beschrieben, besonders wenn ethnische oder andere Kategorisierungen im Spiel sind. Wie schon oben mehrfach ausgeführt, werden diese Unterscheidungen von Politik und Marktkräften, Unternehmenspraktiken und Gemeinschaften getroffen und genutzt. Besonders Frauen und ganz besonders Mütter werden oft als in einer anderen Arbeitsmarktposition gesehen als Männer: Entlassungen, Teilzeitarbeit oder Zeiten von Arbeitslosigkeit werden bei Frauen als weniger problematisch angesehen, ihre Einkommen sind in der Tendenz eher niedrig.

\section{Anwendungsmöglichkeiten}

Der vorliegende Beitrag vertritt einen erweiterten Ansatz zur Untersuchung von Arbeits- und Sozialpolitikregimen - ausgehend von der Annahme, dass Politik und Praxis über einen stabilen Zeitraum hinweg gemeinsam als System koexistieren, sodass sich von einem "Regime“ sprechen lässt. In einigen Fällen wie in Mittel- und Osteuropa findet sich jedoch ein sich ständig veränderndes Spektrum kaum verbundener Elemente. Die Analyse sollte sich daher nicht ausschließlich auf den Staat zum Schutz vor Marktunsicherheiten beschränken, sondern die ganze Breite institutioneller Möglichkeiten von Gemeinschaften und anderen Formen von Governance beachten. Ebenso möglich wie unerlässlich sind Untersuchungen über die Veränderungen innerhalb der Systeme über die Zeit sowie synchrone Vergleiche zwischen den einzelnen Ländern. Hierdurch lassen sich allgemeine Trends erkennen. Beim Blick auf Veränderungen der Dimensionen Zeit, Ort, Verteilung und Grad der Auslagerung werden sich auch Änderungen bei den Governanceformen beobachten lassen.

In einem ersten Schritt der Anwendung dieses Ansatzes ist zu analysieren, welche politischen Gruppierungen und Praktiken für nachhaltige Sicherheit relevant sind, mit welcher Gewichtung diese sich in den einzelnen Ländern finden und wie sie sich über die Zeit verändert haben.

Zweitens ist es erforderlich, die zeitlichen Veränderungen innerhalb der einzelnen Fälle zu untersuchen, da einige Sozialmodelle und Modellkombinationen an Bedeutung verlieren, während andere in den verschiedenen Ländern oder auch allgemein stärker werden. Dadurch lässt sich die Entwicklung der sozialen Modelle innerhalb Europas besser verfolgen. Soweit möglich, muss der Ansatz noch um einen anderen, damit zusammenhängenden Faktor erweitert werden: die Beobachtung, 
wie sich das zeitliche Verhältnis zwischen gegenwärtiger Flexibilität und Sicherheit von morgen entwickelt. So haben z.B. die Rentenreformen der letzten Jahre in vielen Ländern negative Auswirkungen auf die Zukunftserwartungen hinsichtlich sozialer Sicherheit bei den jüngeren Arbeitnehmern gezeigt, vor allen auf Arbeitsmärkten mit großem Anteil an befristeten und prekären Beschäftigungsverhältnissen.

Drittens führen die Untersuchungen darüber, wie die einzelnen Modi von Governance gemeinsam auftreten oder sich gegenseitig „abstoßen“, vielleicht zu einer Theoriebildung über institutionelle Komplementaritäten bzw. Unverträglichkeiten: Die Bewertung der Verbindungen verschiedener Governancemodi und der dabei beobachtbaren Unterschiede von Klassen- und anderem Interessensausgleich könnte so mit der Entwicklung von Politik und Praxis in Beziehung gebracht werden. So hat z.B. die geringe Jobsicherheit in Dänemark in Verbindung mit einem starken Sozialstaat, starken Gewerkschaf- ten, speziellen Nischenexportprodukten und industriellen Zentren ganz andere Effekte als in Ländern ohne diese Charakteristika.

Die unterschiedlichen GovernanceModi in den verschiedenen Politik- und Praxisfeldern stellen eine Herausforderung dar. Die Krise des Keynesianismus wurde oft als Krise der Verbände (oder des Neokorporatismus) angesehen. Der Verlass auf die Steuerungsmechanismen der Marktwirtschaft erschien als umso vorteilhafter (oftmals unterstützt durch starke staatliche Interventionen). Seither scheint die politische Aktivität großer Einzelunternehmen die der Verbände und des Staates bei der Differenzierung zwischen Arbeitnehmerkategorien und -rechten, bei Lohn- und Rentenfindung ersetzt zu haben. Die Thematik öffentlicher Güter, die sich mit sozialer Ungewissheit und Umweltzerstörung verbindet, wirft erneut die Frage nach der Angemessenheit marktund einzelwirtschaftlicher Governance auf. In diesen Zusammenhängen wird die
Notwendigkeit der Suche nach neuen Formen oder neuen Kombinationen der alten Formen, deutlich sichtbar.

Es ist daher an der Zeit für Forschung und Politik, über den Fokus auf Flexicurity hinauszublicken und die Frage einer nachhaltigen Produktion gesellschaftlicher Sicherheit genereller anzugehen. Die Flexibilität der Arbeit ist nicht das einzige wichtige Thema im wirtschaftlichen Wandel; es gibt nicht notwendigerweise einen trade-off zwischen Sicherheit und Flexibilität, explizite Arbeits- und Sozialpolitik sind nicht die einzigen Bereiche, auf denen nachhaltige Sicherheit erreicht werden kann. Die zentrale Forschungsaufgabe ist daher, verschiedene Formen von Politik und Praxis zu untersuchen, um zu entdecken, mit welchen Mitteln dort Schutz und Sicherheit für die unterschiedlichsten Personenkreise hergestellt wird und werden kann.

\section{LITERATUR}

Bellofiore, R./Halevi, J. (2009): Deconstructing Labor. A MarxianKaleckian perspective on what is "new" in contemporary capitalism and economic, Konferenzpapier Congrès Marx International V, Oktober 2007, Paris, http://actuelmarx.u-paris10.fr/cm5/com/MI5_Eco_Bellofiore_Halevi.doc

Blanchard, O. J./Summers, L. H. (1986): Hysteresis and the European Unemployment Problem, in: NBER Macroeconomics Annual 1, S. 15-78 Buehn, A./Schneider, F. (2007): Shadow Economies and Corruption All Over the World: Revised Estimates for 120 Countries, in: Economics: The Open-Access, Open-Assessment E-Journal 1 (9), http://dx.doi. org/10.5018/economics-ejournal.ja.2007-9

Coase, R. (1937): The Nature of the Firm, in: Economica 4 (16),

S. 386-405

Crouch, C. (2006): Capitalist Diversity and Change, Oxford

Crouch, C. (2009): Privatised Keynesianism: an unacknowledged policy regime, in: British Journal of Politics and International Relations 11 (3),

S. 382-399

Davies, P./Freedland, M. (2007): Towards a Flexible Labour Market: Labour Legislation and Regulation since the 1990s, Oxford

Esping-Andersen, G. (1999): The Social Foundations of Post-Industrial Economies, Oxford

Fama, E. F. (1971): Risk, Return and Equilibrium, in: Journal of Political Economy 79 (1), S. 30-55

Fama, E. F. (1991): Efficient Capital Markets II, in: Journal of Finance 46 (5), S. 1575-1617
Hollingsworth, J. R./Boyer, R. (1997) (Hrsg.): Contemporary Capitalism: The Embeddedness of Institutions, Cambridge

Hollingsworth, J. R./Müller, K. H./Hollingsworth, E. J. (2002):

Advancing Socio-Economics: An Institutionalist Perspective, Lanham Jurado Guerrero, T. (1999): Why Do Spanish Young People Stay Longer at Home than the French? The Role of Employment, Housing and Social Policies, Florenz (unveröffentlichte PhD thesis)

Kenney, M. (2000): Understanding Silicon Valley: The Anatomy of an Entrepreneurial Region, Stanford

Knegt, R. (Hrsg.) (2008): The Employment Contract as an Exclusionary Device. An Analysis on the Basis of 25 Years of Developments in the Netherlands, Antwerpen

Kristensen, P. H./Karnøe, P./Andersen, P. H. (Hrsg.) (1999): Mobilizing Resources and Generating Competencies. The remarkable success of small and medium sized enterprises in the Danish business system, Kopenhagen

Majone, G. (1990): Deregulation or Re-regulation? Regulatory Reform in Europe and the United States, London

Muffels, R./Chung, H./Fouarge, D./Klammer, U./Luijkx, R./Manzoni, A./Thiel, A./Wilthagen, T. (2008): Flexibility and security over the life course, Dublin

Olson, M. (1965): The Theory of Collective Action, Cambridge, MA Olson, M. (1982): The Rise and Decline of Nations, New Haven Pyke, F./Beccatini, G./Sengenberger, W. (1990): Industrial Districts and Inter-Firm Co-operation in Italy, Genf 
Rogowski, R. (Hrsg.) (2008): The European Social Model and Transitional Labour Markets: Law and Policy, Aldershot

Rueda, D. (2005): Insider-Outsider Politics in Industrialized Democracies: the Challenge to Social Democratic Parties, in: American Political Science Review 99 (1), S. 61-74

Rueda, D. (2007): Social Democracy Inside Out. Partisanship and Labor Market Policy in Industrialized Democracies, Oxford

Schmitter, P. C./Streeck, W. (Hrsg.) (1985): Private Interest Government: Beyond Market and State, London

Stinchcombe, A. L. (1965): Social structure and organizations, in: March, J. G. (Hrsg.): Handbook of Organization, Chicago, IL, S. $142-93$

Talani, L. S./Cerviño, E. (2003), Mediterranean Labour and the Impact of Economic and Monetary Union: Mass Unemployment or Labour Market Flexibility?, in: Overbeek, H. (Hrsg.): The Political Economy of European Employment, London, S. 199-226

Traxler, F. (2003): Bargaining, (de)centralization, macroeconomic performance and control over the employment relationship, in: British Journal of Industrial Relations 41 (1), S. 1-27

Traxler, F./Blaschke, S./Kittel, B. (2001): National Labour Relations in Internationalized Markets, Oxford
Traxler, F./Brandl, B./Glassner, V. (2008): Pattern Bargaining: an Investigation into its Agency, Context and Evidence, in: British Journal of Industrial Relations 46 (1), S. 33-58

Wallerstein, I. (1986): Das moderne Weltsystem, Bd. 1: Die Anfänge kapitalistischer Landwirtschaft und die europäische Weltökonomie, Frankfurt a. M.

Williamson, O. E. (1975): Markets and Hierarchies: Analysis and Antitrust Implications: A Study in the Economics of Internal Organization, New York

Williamson, O. E. (1985): The Economic Institutions of Capitalism, New York

Wilthagen, T. (2002): Managing Social Risks with Transitional Labour Markets, in: Mosley, H./O'Reilly, J./Schömann, K. (Hrsg.): Labour Markets, Gender and Institutional Change: Essays in Honour of Günther Schmid, Cheltenham et al., S. 264-289

Wilthagen, T./Tros, F. (2004): The Concept of "Flexicurity": a new approach to regulating employment and labour markets, in: Transfer 10 (2), S. 166-186 